\title{
ACEITABILIDADE DO PALMITO DE PUPUNHA
}

\author{
MARTA REGINA VERRUMA-BERNARDI * \\ ANA CAROLINA DUTRA CAVALCANTI ** \\ SHIZUKO KAJISHIMA *
}

\begin{abstract}
Avaliou-se o padrão de consumo de palmito e a aceitabilidade do palmito de pupunha (Bactris gasipaes H.B.K.). Aplicouse questionário a um grupo de 80 indivíduos para avaliar a freqüência e os hábitos de consumo de palmito, segundo a faixa etária. O teste de aceitação do palmito de pupunha foi realizado com 45 julgadores, sendo apresentadas 2 amostras (em rodelas e picadas) na forma de conserva. As avaliações de aceitação foram realizadas utilizando-se escala hedônica de 9 pontos. A porcentagem de aprovação e de rejeição das amostras analisadas ocorreu, respectivamente, nas notas maiores e menores que $5 \mathrm{da}$ escala hedônica. A freqüência de consumo de palmito mostrou que $78 \%$ dos julgadores consomem o produto 1 vez ao mês e $16 \%$ até 4 vezes ao mês. As amostras de palmito de pupunha em rodelas e picadas apresentaram 82 e $78 \%$ de aprovação, respectivamente. Ambas as amostras evidenciaram boa aceitação.
\end{abstract}

PALAVRAS-CHAVE: ANÁLISE SENSORIAL; PALMITO DE PUPUNHA.

\section{INTRODUÇÃO}

A pupunheira (Bactris gasipaes H.B.K.) produz frutos comestíveis de sabor agradável e alto valor nutritivo, destacando-se elevados teores de caroteno, de proteína e de gordura. Essa espécie de palmeira produz, além dos frutos, palmito comestível de ótima qualidade. O potencial da pupunheira para a produção de palmito cultivado é considerado maior que o das palmáceas utilizadas para tal finalidade (ARAÚJO, 1996).

O sucesso da pupunheira como produtora de palmito deve-se às suas características de precocidade, produtividade e adaptação. A produção da planta é contínua durante todo o ano (colheita em 9 meses), o crescimento é rápido com o primeiro corte no segundo ano (precocidade)

* Professoras, Departamento de Nutrição e Dietética, Faculdade de Nutrição, Universidade Federal Fluminense (UFF), Niterói - RJ (e-mail:verruma@bol.com.br).

** Ex-aluna do Curso de Nutrição da Faculdade de Nutrição, Universidade Federal Fluminense (UFF), Niterói, RJ. 
e cultivo perene, não necessitando de reposição anual do plantio. No Brasil é cultivada nos estados da Bahia, Espírito Santo, São Paulo e Rio de Janeiro.

De acordo com VILLACHICA et al. (1994) são retirados dois envoltórios externos do talo colhido, ficando apenas dois envoltórios internos para proteção do palmito. Cerca de $59,6 \%$ do seu peso $( \pm 755 \mathrm{~g})$ é representado pela casca, $14,6 \%$ pela parte basal, $10,7 \%$ pelas folhas abertas ou "ponta" e 15\% pelo palmito aproveitável. O palmito é submetido à ação de calor e envasado em solução à base de sal e ácido cítrico. Análises de palmito enlatado e armazenado por 90 dias mostraram valores similares ao palmito fresco, com pequenas variações devido ao processamento. SOARES (1997) sugere como alternativa de processamento o uso de soluções salinas com imersão do palmito e aplicação de atmosfera controlada.

Para FERREIRA et al. (1990) a constante e crescente necessidade de suprimento de matéria-prima, para as indústrias no mercado nacional e internacional, tem despertado especial interesse em outros gêneros da família Palmae, particularmente os capazes de fornecer palmito de boa qualidade em curto prazo. Segundo ARAÚJO (1996) a pupunha poderá exercer papel importante na solução do problema de oferta de matériaprima no Brasil, considerando a precocidade da produção de palmito com cortes anuais após o primeiro ano, contra 4 a 8 anos para o açaí e 0 juçara, respectivamente.

BERNHARDT (1999) afirmou que a principal característica do palmito da pupunheira, sob o ponto de vista do processamento, é a sua baixa atividade enzimática com conseqüente escurecimento bastante lento. Devido à ausência de enzimas oxidantes polifenoloxidase e peroxidase a oxidação é praticamente nula. Isso facilita o processamento e permite desenvolver outras formas de comercialização do produto, com alterações mínimas no sabor e aroma após o beneficiamento e possibilitando a manutenção de textura firme e macia.

A pupunha apresenta-se como alternativa de cultivo racional no contexto de diminuição de oferta e crescimento de consumo de palmito. Devido ao potencial comercial do palmito de pupunha muitos países latino-americanos estão investindo no seu cultivo e industrialização. O interesse pelo cultivo da pupunha tem aumentando fortemente (VILLACHICA, 1996), especialmente para a produção de palmito. Dois fatores estão facilitando tal aumento, ou seja, a existência de mercado em nível mundial e a disponibilidade de tecnologia para o cultivo e industrialização do palmito de pupunha. 
O objetivo deste trabalho foi avaliar o padrão de consumo de palmito e a aceitabilidade do palmito de pupunha (Bactris gasipaes H.B.K) na forma de conserva, apresentado em dois diferentes cortes.

\section{MATERIAL E MÉTODOS}

\subsection{MATÉRIA-PRIMA}

Foram utilizadas amostras de duas marcas de palmito de pupunha, sendo uma apresentada em corte tipo rodelas e a outra em corte tipo picado. A amostra $\mathrm{A}$ e a $\mathrm{B}$ foram constituídas de palmito de pupunha ecologicamente cultivado, assim considerado pelo rigoroso controle de higiene e qualidade efetuado em todas as etapas de fabricação. São produtos ecológicos, renováveis e não-extrativistas, segundo os fabricantes.

\subsection{CARACTERIZAÇÃO DOS HÁBITOS DE CONSUMO DOS JULGADORES DE PALMITO}

Entregou-se o questionário, apresentado no Quadro 1, para um grupo de 80 indivíduos, constituído de alunos de graduação, professores e funcionários da Universidade Federal Fluminense. Avaliou-se, mediante o referido questionário a freqüência média mensal e os hábitos de consumo referentes aos palmitos comumente comercializados, segundo a faixa etária dos julgadores convidados a participar do experimento. Paralelamente, os julgadores foram informados da data, local e horário de realização dos testes.

\subsection{TESTE SENSORIAL DE ACEITAÇÃO}

O teste de aceitação, com 45 provadores, foi realizado no Laboratório de Alimentos e Dietética da Faculdade de Nutrição da Universidade Federal Fluminense (UFF).

As amostras foram servidas uma de cada vez, em ordem aleatória de apresentação e temperatura ambiente, sendo codificadas com três dígitos diferentes. Cada amostra estava acompanhada de ficha de aceitabilidade, utilizando-se escala hedônica de 9 pontos para sua avaliação (Quadro 2). Na mesma ficha constavam perguntas sobre o que o julgador mais gostou ou desgostou na amostra de acordo com a metodologia utilizada por BEHRENS et al. (1999). 


\section{QUADRO 1 - QUESTIONÁRIO DE CARACTERIZAÇÃO DOS HÁBITOS DE CONSUMO DOS JULGADORES}

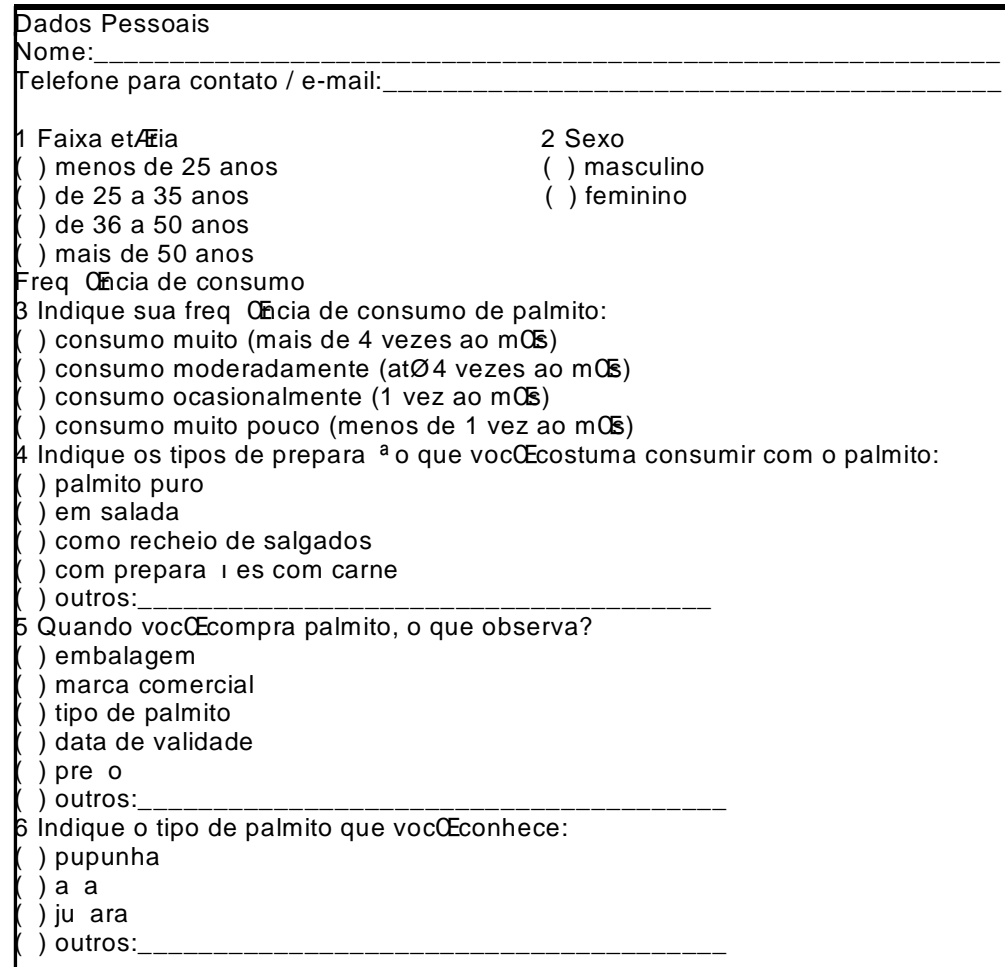

\section{QUADRO 2 - FICHA UTILIZADA PARA AVALIAÇÃO DA ACEITABILIDADE DAS AMOSTRAS DE PALMITO DE PUPUNHA}

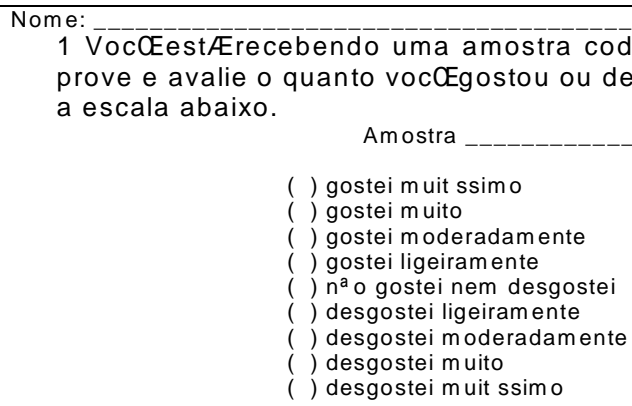
a escala abaixo.

Amostra prove e avalie o quanto vocCEgostou ou desgostou da mesma utilizando

2 Cite o que vocCEmais gostou na amostra:

3 Cite o que vocCEmenos gostou na amostra: 
Com base nos resultados obtidos foram construídos histogramas de freqüência com os valores recebidos pelos dois tipos de amostra.

\section{RESULTADOS E DISCUSSÃO}

\subsection{CARACTERIZAÇÃO DOS HÁBITOS DE CONSUMO DE PALMITO}

Os alunos de graduação representaram $86 \%$ dos 80 indivíduos que responderam o questionário, os professores $10 \%$ e os funcionários $4 \%$. Desses, $82 \%$ enquadraram-se na faixa etária de 25 anos ou menos e $18 \%$ entre 25 a 50 anos, sendo $88 \%$ do sexo feminino e $12 \%$ do sexo masculino.

A freqüência de consumo (Figura 1) revelou que $54,3 \%$ do total de julgadores consomem muito pouco palmito.

\section{FIGURA 1 - FREQÜÊNCIA DE CONSUMO DE PALMITO REPORTADA PELOS JULGADORES}

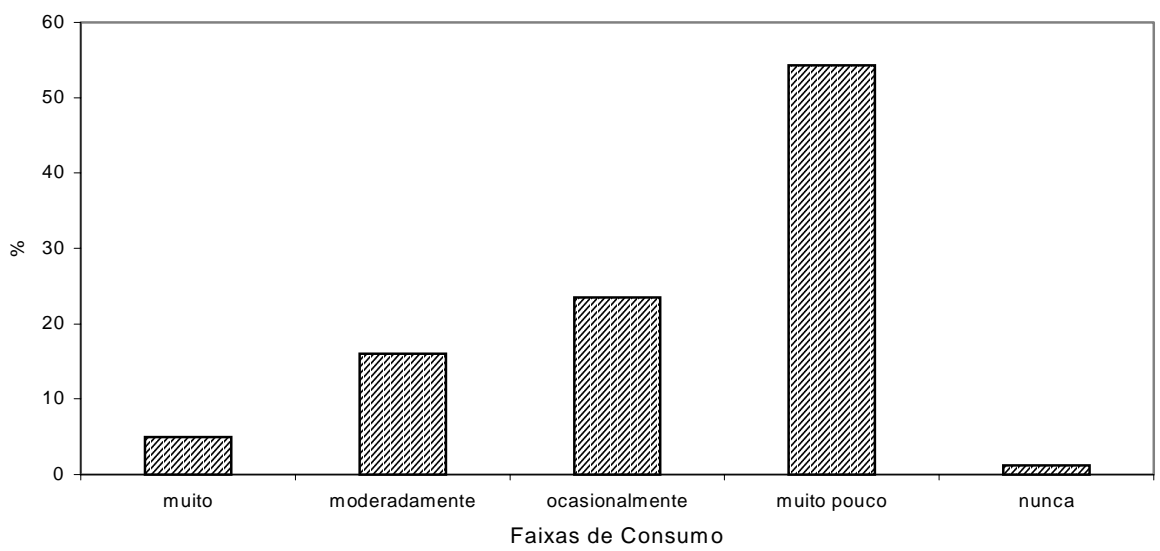

Muito $=$ mais de 4 vezes ao mês; Moderadamente $=$ até 4 vezes ao mês; Ocasionalmente $=1$ vez ao mês; Muito pouco $=$ menos de 1 vez ao mês.

Quanto aos tipos de preparo do palmito (Figura 2) que costumam consumir, $81,5 \%$ declararam consumi-lo em salada e 50,6\% como recheio de salgados. Preparações com carne foram indicadas por $13,6 \%$ dos julgadores e palmito puro por apenas $12,4 \%$. Preparações como estrogonofe e pizza foram pouco citadas. 
FIGURA 2 - FORMA DE CONSUMO DO PALMITO

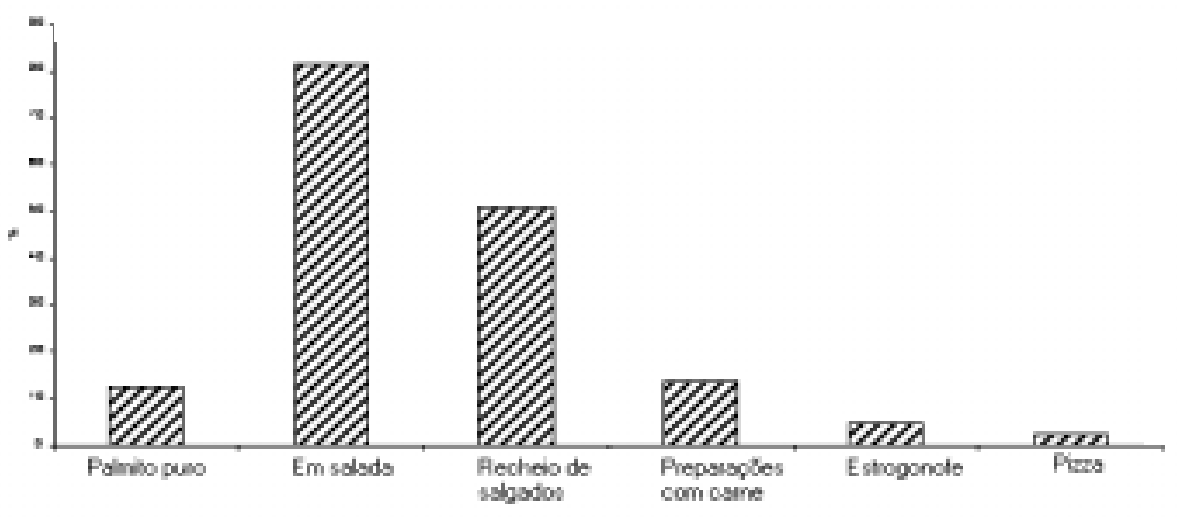

Formas de Consumo

Em relação ao que é observado no ato da compra (Figura 3), 88,9\% dos julgadores citaram a data de validade como prioridade, 59,3\% declararam verificar o tipo de embalagem e $45,6 \%$ ressaltaram o preço. A marca comercial e o tipo de palmito também foram citados por $35,8 \%$ e $33,3 \%$, respectivamente, do total de julgadores. Pequena porcentagem $(1,2 \%)$ de julgadores preocupa-se em observar o selo de garantia.

FIGURA 3 - ITENS OBSERVADOS PELOS ENTREVISTADOS NO ATO DA COMPRA

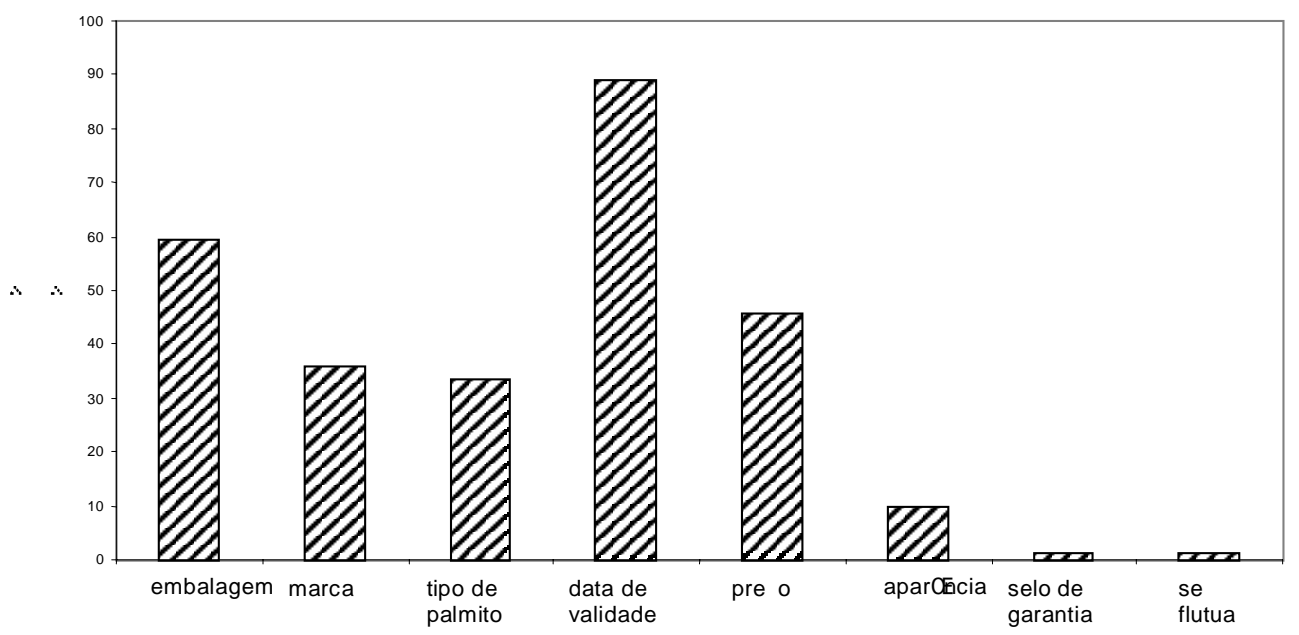

126

B.CEPPA, Curitiba, v. 21, n. 1, jan./jun. 2003 
O tipo de palmito mais conhecido foi o juçara (29,6\%), seguido pelo açaí $(27,2)$ e pelo palmito de pupunha $(16,1 \%)$, sendo que $39,5 \%$ dos julgadores não souberam responder essa pergunta (Figura 4).

\section{FIGURA 4 - TIPOS DE PALMITO MAIS CONHECIDOS PELOS JULGADORES}

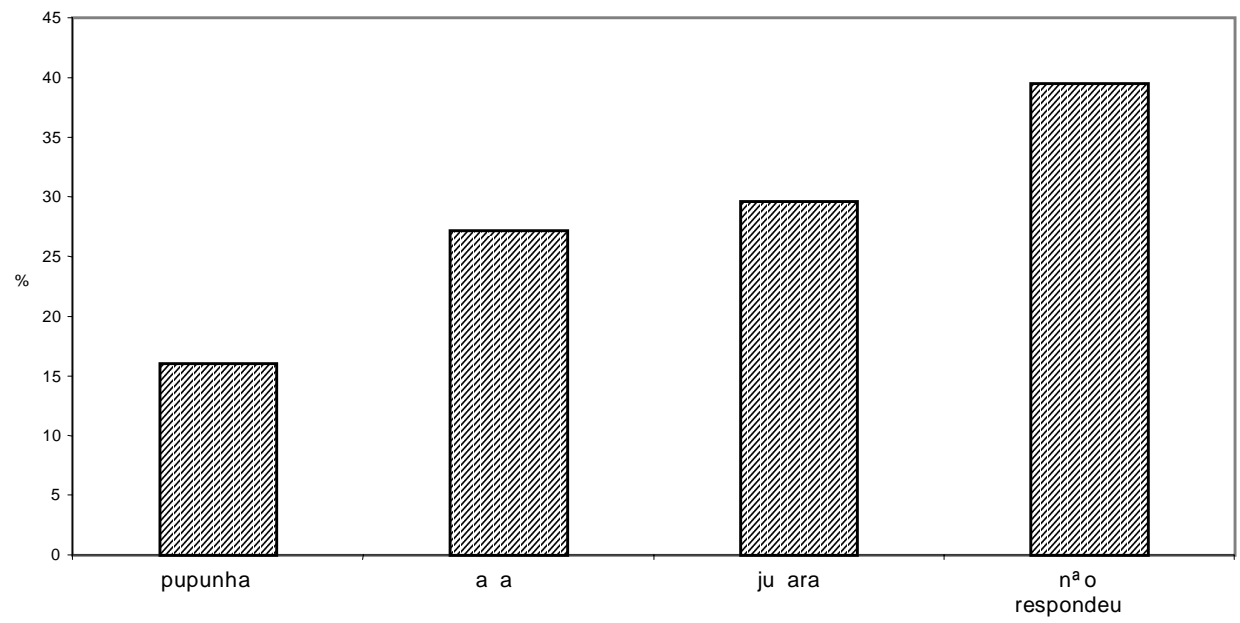

3.2 ACEITAÇÃO DO PALMITO DE PUPUNHA

A Tabela 1 apresenta a porcentagem de aprovação e de rejeição das amostras analisadas, sendo que ambas obtiveram alto índice de aceitação.

TABELA 1 - ACEITAÇÃO DAS AMOSTRAS DE PALMITO DE PUPUNHA

\begin{tabular}{cccc}
\hline Amostras & MØdias & $\begin{array}{c}\text { Aprova } \\
(\%)\end{array}$ & $\begin{array}{c}\text { Rejei }^{a} 0^{*} 0^{* *} \\
(\%)\end{array}$ \\
\hline A & 6,6 & 82,22 & 17,78 \\
B & 5,9 & 77,78 & 22,22 \\
\hline
\end{tabular}

*notas superiores a 5 .

${ }^{\star *}$ notas inferiores a $5 .(1=$ desgostei muitíssimo $/ 9$ = gostei muitíssimo $)$.

A amostra A (palmito em rodelas) obteve $82,2 \%$ de aprovação contra $17,8 \%$ de rejeição. Aproximadamente $31,2 \%$ dos julgadores gostaram moderadamente dessa amostra e 24,4\% gostaram muito. Apenas 13,3\% dos julgadores assinalaram a categoria "desgostei ligeiramente" na escala hedônica. 
A amostra B (palmito picado) obteve $77,8 \%$ de aprovação e $22,2 \%$ de rejeição. A maioria dos julgadores gostou ligeiramente da amostra $\mathrm{B}$ e $17,8 \%$ gostaram moderadamente.

FERREIRA et al. (1982) estudaram a aceitabilidade do palmito de pupunha em conserva em relação ao palmito juçara e constataram boa aceitação. GOMES (1996) verificou que o palmito de pupunha apresenta sabor agradável e superioridade em relação ao palmito de açaí em termos de calorias e teores de proteínas, lipídios e glicídios. MORAES (2003) constatou tendência para sabor amargo, mediante análise sensorial descritiva do palmito de pupunha. CLEMENT et al. (1999) estudaram a qualidade sensorial do palmito ao longo de sua conservação sob refrigeração. Verificaram que o amargor aumentou significativamente no tratamento com absorvente de oxigênio, especialmente a partir do $18^{\circ}$ dia, sendo a principal característica responsável pela rejeição do palmito.

Os resultados da Tabela 2 evidenciam que o atributo textura foi o que mais agradou aos julgadores (53,3\% para amostra A e 54,5\% para a amostra B). Os julgadores citaram o sabor amargo como característica negativa da amostra $B(14,29 \%$ dos julgadores).

\section{TABELA 2 - CARACTERÍSTICAS SENSORIAIS MAIS APRECIADAS E MENOS APRECIADAS PELOS JULGADORES EM RELAÇÃO ÀS AMOSTRAS A E B}

\begin{tabular}{c|c|c|c|c}
\hline Amostras & $\begin{array}{c}\text { Caracter sticas } \\
\text { mais apreciadas }\end{array}$ & \% das respostas & $\begin{array}{c}\text { Caracter sticas } \\
\text { menos } \\
\text { apreciadas }\end{array}$ & $\%$ das respostas \\
\hline A & Textura & 53,4 & Textura & 26,5 \\
& Sabor & 42,2 & Sabor & 44,1 \\
& Aroma & 4,4 & Cor & 23,5 \\
& Aroma & 5,9 \\
\hline & Textura & 54,5 & Textura & 21,5 \\
& Sabor & 31,8 & Sabor & 47,6 \\
& AparCficia & 11,4 & AparCfiacia & 7,1 \\
& Aroma & 2,3 & Aroma & 9,5 \\
& & & Sabor amargo & 14,3 \\
\hline
\end{tabular}

Em estudo realizado por FERREIRA et al. (1982) foi constatado que o palmito de pupunha apresenta excelente aparência. Os toletes são bem formados e opacos, de cor amarelo-clara uniforme. A textura é mais firme, o sabor lembra gosto de "mato" com ligeiro amargor no final. Para SOARES 
(1997) e MORAES (2003) a pupunha é uma espécie de palmito que apresenta características próprias e muito distintas, tais como textura macia e sabor ligeiramente adocicado.

\title{
4 CONCLUSÃO
}

A freqüência de consumo de palmito mostrou que $78 \%$ dos julgadores consomem o produto 1 vez ao mês e $16 \%$ até 4 vezes ao mês.

As amostras de palmito de pupunha em rodelas e picadas apresentaram $82 \%$ e $78 \%$ de aprovação, respectivamente. Ambas as amostras evidenciaram tendência de boa aceitação, sendo a textura a característica mais apreciada pelos julgadores. O sabor foi menos apreciado devido ao ligeiro amargor no final.

\begin{abstract}
ACCEPTANCE OF PEJIBAYE PALM HEART

It was evaluated the pattern of consumption of palm heart and the acceptance of pejibaye palm heart (Bactris gasipaes H.B.K.). A questionnaire was applied to a 80members group, to evaluate, the frequency and habits of consumption of the palm heart according to the age group. The acceptance test of pejibaye palm heart was accomplished with 45 judges, being presented two samples (sliced and pricked) of pejibaye palm heart in preserve. The evaluations were made using a 9-points hedonic scale. The percentage of approval and rejection of the samples occurred when the scores were respectively higher and lower than 5 in the hedonic scale. The frequency of consumption of palm heart showed that $78 \%$ of the total judges eat it once a month; and $16 \%$, until 4 times in a month. The slice and pricked samples obtained 82 and $78 \%$ of approval, respectively. The results showed that both samples obtained the same tendency of good acceptance indexes.
\end{abstract}

KEY-WORDS: SENSORIAL ANALYSIS; PEJIBAYE PALM HEART.

\section{REFERÊNCIAS}

1 ARAÚJO, I.C. Potencialidade da pupunheira: uma visão do ponto de vista do agribusiness. In: WORKSHOP SOBRE AS CULTURAS DE CUPUAÇU E PUPUNHA NA AMAZÔNIA, 1, 1996, Manaus. Anais... Manaus: EMBRAPA-CPAA, 1996.

2 BERNHARDT, L.W. Características do palmito da pupunheira do ponto de vista do processamento. In: SEMINÁRIO DO AGRONEGOCIO, 1999, Porto Velho. Palmito de pupunha na Amazônia. Porto Velho: Embrapa Rondônia/Sebrae-RO, 1999. p. 24-33. 
3 BEHRENS, J.H.; SILVA, M.A.A.P da.; WAKELING, I.N. Avaliação da aceitação de vinhos brancos varietais brasileiros através de testes sensoriais afetivos e técnica multivariada de mapa de preferência interno. Revista da Sociedade Brasileira de Tecnologia de Alimentos, v.19, n.2, p.214-220, 1999.

4 CLEMENT, C.R.; SANTOS, L.A.; ANDRADE, J.S. Conservação de palmito de pupunha em atmosfera modificada. Acta Amazonica, v.29, n.3, p. 437-445, 1999.

5 FERREIRA, V.L.P.; GRANER, M.; BOVI, M.L.A.; DRAETTA, I.S.; PASCHOALINO, J.E.; SHIROSE, I. Comparação entre os palmitos de (Guilielma gasipaes Bailey) (pupunha) e Euterpe edulis Mart. (juçara) I. Avaliações físicas, organolépticas e bioquímicas. Coletânea do Instituto de Tecnologia de Alimentos, v.12, p.255-272, 1982.

6 FERREIRA, V.L.P.; GRANER, M.; BOVI, M.L.A.; FIGUEIREDO, I.B.; ANGELUCCI, E.E; YOKOMIZO, Y. Comparação entre os palmitos das palmeiras Guilielma gasipaes Bailey (pupunha) e Euterpe edulis Mart. (juçara). II. Avaliações físicas e químicas. Coletânea do Instituto de Tecnologia de Alimentos, v.12, p.273-282, 1981/ 1982.

7 FERREIRA, V. L. P. et al. Composição química e curvas de titulação de acidez do palmito de pupunha (Bactris gasipaes H.B.K.) de diversas localidades. Coletânea do Instituto de Tecnologia de Alimentos, v.20, p.96-104, 1990.

8 GOMES, J.B.M. Pupunheira: uma planta alternativa para produção racional de palmito. Informativo da Sociedade Brasileira de Fruticultura, v.5, n.3, p.1416, 1996.

9 MORAES, C.W.P. Análise sensorial descritiva do palmito de pupunha. Rio de Janeiro: Faculdade de Nutrição/UFF, 2003. 35 p. (Trabalho Final de Curso).

10 SOARES, A.G. Palmito de pupunha: alternativas de processamento. Revista de Horticultura Brasileira, v.15, p.198-199, 1997.

11 VILLACHICA, H.; CHÁVEZ, E.; SANCHEZ, J. Manejo post cosecha e industrialización del pijuayo (Bactris gasipaes Kunth.). Informe Técnico, n.30, p.55, 1994.

12 VILLACHICA, H. Cultivo del pijuayo (Bactris gasipaes Kunth.) para palmito en la Amazonia. Lima, Peru: FAO/Tratado de Cooperacion Amazonica, 1996. $153 \mathrm{p}$. 